Ferrata Storti Foundation

\title{
TIRAP p.R81C is a novel lymphoma risk variant which enhances cell proliferation via NF-kB mediated signaling in B-cells
}

Haematologica 2019

Volume 104(4):766-777

\section{Correspondence: \\ URBAN NOVAK \\ urban.novak@insel.ch \\ Received: August 9, 2018. \\ Accepted: October 30, 2018. \\ Pre-published: October 31, 2018.}

doi:10.3324/haematol.2018.201590

Check the online version for the most updated information on this article, online supplements, and information on authorship \& disclosures: www.haematologica.org/content/104/4/766

\section{(C)2019 Ferrata Storti Foundation}

Material published in Haematologica is covered by copyright. All rights are reserved to the Ferrata Storti Foundation. Use of published material is allowed under the following terms and conditions:

https://creativecommons.org/licenses/by-nc/4.0/legalcode. Copies of published material are allowed for personal or internal use. Sharing published material for non-commercial purposes is subject to the following conditions:

https://creativecommons.org/licenses/by-nc/4.0/leǵalcode, sect. 3. Reproducing and sharing published material for commercial purposes is not allowed without permission in writing from the publisher.

\author{
Regula Burkhard, ${ }^{1,2,3}$ Irene Keller, ${ }^{4}$ Miroslav Arambasic, ${ }^{1,2,3}$ \\ Darius Juskevicius, ${ }^{5}$ Alexandar Tzankov, ${ }^{5}$ Pontus Lundberg, ${ }^{6}$ \\ Rémy Bruggmann, ${ }^{4}$ Stephan Dirnhofer, ${ }^{5}$ Ramin Radpour ${ }^{1,7^{*}}$ \\ and Urban Novak ${ }^{1,3^{*}}$
}

${ }^{1}$ Department of Medical Oncology, Inselspital, Bern University Hospital; ${ }^{2}$ Division of Experimental Pathology, Institute of Pathology, University of Bern; ${ }^{3}$ Department for BioMedical Research (DBMR), University of Bern; ${ }^{4}$ Interfaculty Bioinformatics Unit, Department for BioMedical Research, and Swiss Institute of Bioinformatics, University of Bern; ${ }^{5}$ Institute of Pathology and Medical Genetics, University of Basel; ' Department of Biomedicine, Experimental Hematology, University Hospital Basel and University of Basel and ${ }^{7}$ Tumor Immunology, Department for BioMedical Research (DBMR), University of Bern, Switzerland

${ }^{\star} R R$ and UN are co-senior authors.

\section{ABSTRACT}

iffuse large B-cell lymphoma is the most common malignant lymphoma in adults. By gene-expression profiling, this lymphoma is divided in three cell-of-origin subtypes with distinct molecular and clinical features. Most lymphomas arise sporadically, yet familial clustering is known, suggesting a genetic contribution to disease risk. Familial lymphoma cases are a valuable tool to investigate risk genes. We studied a Swiss/Japanese family with 2 sisters affected by a primary mediastinal B-cell lymphoma and a non-germinal center diffuse large B-cell lymphoma not otherwise specified, respectively. The somatic landscape of both lymphomas was marked by alterations affecting multiple components of the JAK-STAT pathway. Consequently, this pathway was constitutively activated as evidenced by high pJAK2 as well as increased nuclear pSTAT3 and pSTAT6 in malignant cells. Potential lymphoma risk variants were identified by whole exome sequencing of the germline DNA derived from siblings and unaffected family members. This analysis revealed a pathogenic variant in TIRAP, an upstream regulator of NF- $\kappa B$, in both affected siblings and their mother. We observed increased B-cell proliferation in family members harboring the TIRAP $p$. R81C variant. B-cell proliferation correlated with

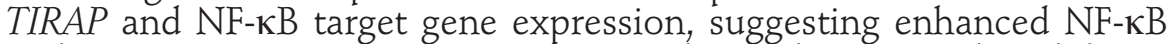
pathway activity in TIRAP p.R81C individuals. TIRAP knockdown reduced B-cell survival and NF- $\mathrm{BB}$ target gene expression, particularly in individuals with TIRAP p.R81C. Functional studies revealed significantly increased NF- $\mathrm{KB}$ activity and resistance to stress-induced cell-death by TIRAP p.R81C. The identification of an inherited TIRAP variant provides evidence for a novel link between genetic alterations affecting the NF-кB pathway and lymphomagenesis.

\section{Introduction}

Diffuse large B-cell lymphoma (DLBCL) is the most common lymphoma in adults. ${ }^{1}$ Its molecular subtypes, activated B-cell-like $(A B C)$, germinal center B-celllike (GCB) DLBCL, and primary mediastinal B-cell lymphoma (PMBL) arise from B-cells at distinct differentiation stages. ${ }^{2,3} \mathrm{PMBL}$ is clinically aggressive with bulky mediastinal masses; it accounts for up to $10 \%$ of DLBCLs, and preferentially occurs in young female patients.

Next-generation sequencing provided insights in genetic lesions of de novo DLBCL and its subtypes. ${ }^{4-10}$ The genetic hallmarks of PBML are amplifications of the 9p24 locus containing JAK2 and PDL1. Present in 70\% of PMBL, this amplification is rare in other DLBCL subtypes ${ }^{11-13}$ Constitutive NF-kB pathway activity through various mechanisms is characteristic of PMBL and ABC-DLBCL..$^{14}$

Until now, the genetic risk factors for DLBCL/PMBLs have remained obscure. 
Population-based studies reported an increased risk for DLBCL in relatives of individuals with DLBCL, and genome-wide association studies identified several common single nucleotide variants associated with sporadic DLBCL. ${ }^{15-18}$ Familial clustering provides evidence for Mendelian susceptibility. In very rare cases, familial aggregation is associated with hereditary cancer syndromes, ${ }^{18}$ but as far as other syndromes are concerned, a heritable basis for DLBCL is not fully understood. A germline variant in MLL described in a Finnish family is still the only reported variant linked to familial PMBL. ${ }^{19}$ Although familial lymphomas account for less than $5 \%$ of cases, these pedigrees are a valuable tool to help identify risk genes that might also contribute to a better understanding of more frequent sporadic cases.

Here, we investigate a Swiss/Japanese family in which 2 out of 3 children were diagnosed with aggressive B-cell lymphomas arising in the mediastinum. Whole exome sequencing (WES) on the germline DNA of the affected siblings and healthy family members identified a variant in the TIR-domain-containing adaptor protein (TIRAP). TIRAP engages signals from TLR2 and TLR4 receptors and recruits MyD88 to the plasma membrane mediated through Toll/interleukin-1 receptor (TIR) domain interaction. ${ }^{20}$ Downstream signaling includes activation of IL-1R-associated kinases (IRAK), ultimately culminating in the activation of the transcription factors NF- $\mathrm{KB}$ and AP-1. In this family, we identified an inherited TIRAP p.R81C variant in 2 affected siblings. This variant provided B-cells with increased proliferation and survival through enhanced NF- $\mathrm{kB}$ activity. Functional studies revealed that TIRAP $p . R 81 C$ enhanced NF- $\mathrm{KB}$ gene signature and reduced stress-triggered cell death. Collectively, we provide evidence that TIRAP p.R81C may act as a novel lymphoma risk variant and our data suggest that TIRAP should be integrated into the complex network of genes contributing to deregulated NF- $\mathrm{\kappa B}$ signaling involved in lymphomagenesis.

\section{Methods}

\section{Patients}

Samples from patients, non-affected family members, and healthy donors were collected after informed consent. This study was approved by the local ethics committee (KEK-BE116/11) and was conducted in accordance with the Declaration of Helsinki. The diagnosis of DLBCL/PMBL was made according to the 2017 World Health Organization classification and pathological review by SD and AT confirmed the diagnosis. ${ }^{1}$ Genomic DNA was extracted from peripheral blood mononuclear cells (PBMCs) using standard methods. Tumor DNA was isolated from formalin-fixed paraffin embedded tissues (FFPE) using phenol-chloroform. In FFPE samples, tumor cell-rich areas were identified on CD20 stained sections and separated from surrounding tissue by laser microdissection.

\section{Whole exome sequencing}

The quality of extracted DNA was assessed by Bioanalyzer (Agilent) and a PCR fragment size-based assay developed at Fasteris (Geneva, Switzerland). Prior to library preparation with TruSeq DNA Sample Preparation Kit (Illumina), DNA samples were treated with PreCR Repair Mix (New England Biolabs). Exome capturing was performed using TruSeq Exome Enrichment Kit (Illumina) and samples were sequenced on an Illumina
HiSeq2500 instrument with $100 \mathrm{bp}$ paired-end reads (Fasteris, sequencing performed between 2012 and 2014). As the FFPE lymphoma sample of sister 2 resulted in a low-yield library of poor quality, the library preparation was modified: after PreCR Repair mix treatment, DNA was split, and four libraries were prepared simultaneously using the Nextera Exome Enrichment Kit (Illumina). Before exome enrichment, libraries were pooled and sequencing was performed as described above. Both exome enrichment kits contained the same set of baits, resulting in identical exome coverage. Whole exome sequencing (WES) data has been deposited at the European Nucleotide Archive (http://www.ebi.ac.uk/ena, accession number PRJEB15254). See Online Supplementary Table S3 for exome data metrics.

\section{Analysis of germline and somatic variants}

Raw sequence read quality was assessed using FastQC. Reads were mapped to the human reference genome hg19 using Bowtie2 v.2.2.1, and duplicated reads were removed by Pi-card-tools v.1.80. Germline variant calling was performed using the Genome Analysis Toolkit (GATK v.3.3.0) best practices workflow using Haplotype Caller and limiting the analysis to enriched targets $\pm 100 \mathrm{bp}$. We used GATK v.3.3.0 to recalibrate the variant quality and refine the genotypes using population $(1000$ Genomes Project, phase 1 data) and pedigree information. Variants in low complexity regions were removed. ${ }^{21}$ Germline variants were prioritized as following: 1) good quality genotype in both sisters (Phred quality 220); 2) moderate/high impact based on SnpEff v.3.2 prediction; 3) novel/known variant at frequency of less than $1 \%$ (not polymorphisms) according to the 1000 Genomes Database and the Exome Variant Server; and 4) the presence of at least one copy of the putatively harmful allele in both siblings. Only variants not present as homozygote in healthy family members were selected. Possible links between genes with germline variants and terms related to cancer and malignant lymphomas were assessed by Ariadne Genomics Pathway Studio v.9 (Elsevier). Alterations with a predicted link to the disease were annotated with PolyPhen-2, SIFT, MutationTaster and GERP++ effect prediction scores using dbNSFP v.2.1, and Combined Annotation Dependent Depletion (CADD) scores v.1.1 (available from https://cadd. gs.washington.edu/). Pathway Studio was used to identify gene networks and canonical pathway enriched for genes containing putatively deleterious variants. The enrichment-scores were calculated using $\chi^{2}$ test comparing genes with putatively deleterious mutations to the proportion of background genes in the Gene Ontology group. An enrichment-score $\geq 3$ corresponded to a significant link $(P<0.05)$. See also Online Supplementary Appendix.

\section{Results}

\section{Clinical and histopathological characterization of the mediastinal B-cell lymphomas}

The Swiss/Japanese family investigated includes 2 female siblings with lymphomas (Figure 1). The older sister 1 developed a PMBL at 30 years of age and died with primary progressive disease (Online Supplementary Table S1). At 25 years of age, sister 2 was diagnosed with a stage IIA non-germinal center (GC) DLBCL, not otherwise specified (NOS) with a mediastinal mass and cervical lymphadenopathy. Chemo-immunotherapy achieved an ongoing remission. Smoldering myelomas $\operatorname{IgG} \lambda$ were detected in the father and his monozygotic twin at 65 years of age. Other family members are currently healthy, and there are no other hematologic malignancies in the extended family. 


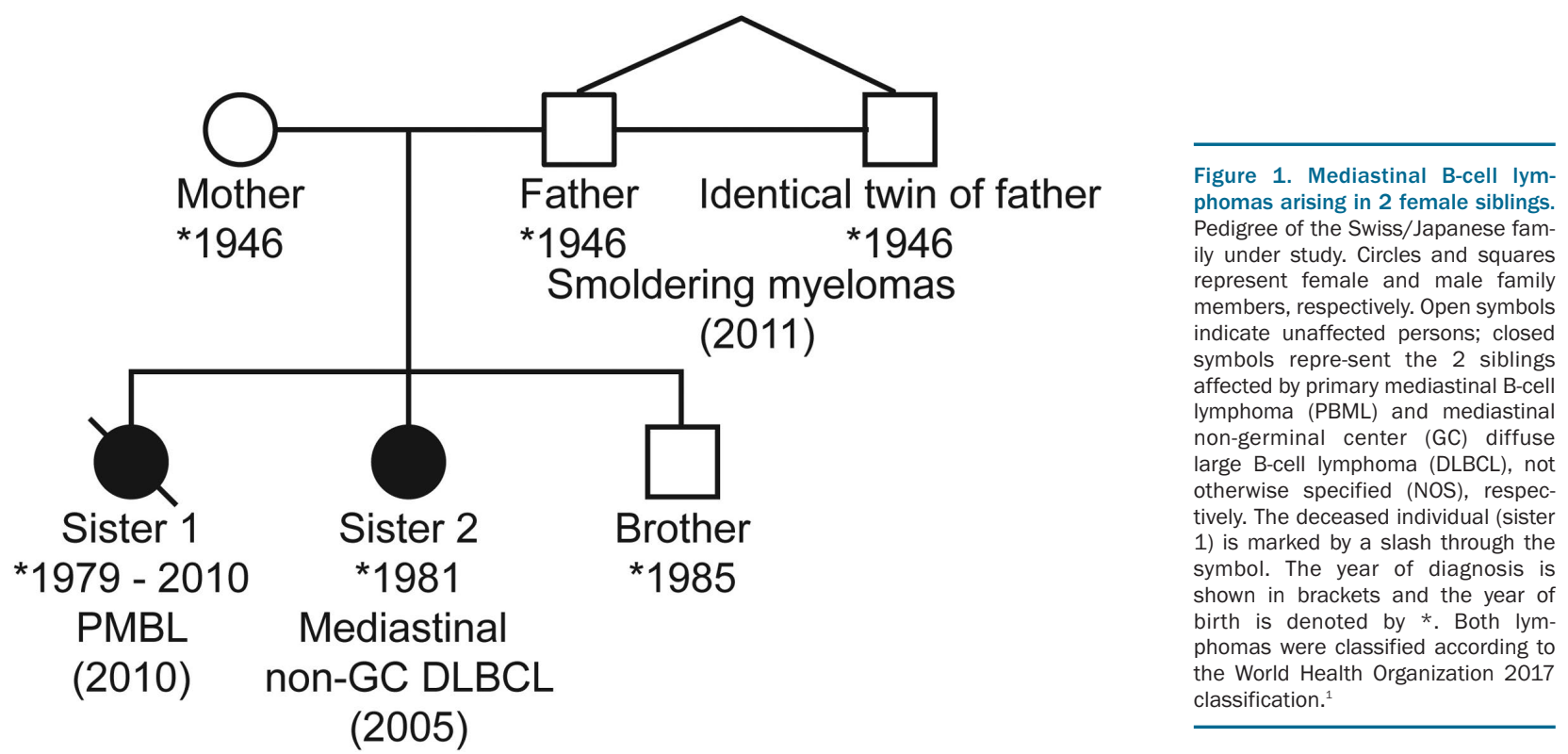

Both lymphomas lacked evidence of an Epstein-Barr virus infection (Online Supplementary Table S2), showed a clear cytoplasm and compartmentalizing sclerosis, and were CD20, CD30 and Ki-67 positive. However, the GC markers BCL6, CD10 and GCET1 were only expressed in the PMBL of sister 1, as was CD23 and BCL2. Despite the expression of $\mathrm{CD} 30$ and sclerosis, the clinical presentation, morphology and immunophenotype of the tumor in sister 2 were consistent with a non-GC DLBCL NOS. ${ }^{1}$ Given its genetic (9p24 and 12q13 gains, SOCS1 and STAT6 mutations), and phenotypic characteristics (expression of CD30, overexpression of JAK2-STAT-cascade members), this lymphoma can retrospectively be considered to most probably represent a PMBL, and was initially designated as non-GC DLBCL NOS with features of PMBL.

\section{Analysis of the coding genome of lymphomas}

The somatic landscape of both lymphomas was analyzed by WES using the Illumina technology on DNA derived from laser-dissected FFPE tissue sections (Online Supplementary Methods). Mutations were validated using Ion Proton sequencing, and their somatic origin was confirmed by the absence in matched normal DNA isolated from PBMCs. A total of 192 and 130 confirmed clonal protein altering mutations were identified in the lymphomas of sisters 1 and 2, respectively (Online Supplementary Table S7). Most of those mutations were missense mutations, with a low number of nonsense and splice site variants (Figure 2A).

In addition, somatic copy number alterations (CNA) were analyzed by array comparative genomic hybridization $(\mathrm{aCGH})$. While the tumor of sister 1 contained seven gains and two deletions, three gains were detected in the lymphoma of sister 2. Interestingly, 9p24 and 12q13 gains were present in both lymphomas (Figure 2B). Fluorescence in situ hybridization (FISH) analyses revealed a trisomy at 8q24 (including MYC) in the lymphoma of sister 1 , in line with the gain on chromosome 8 by aCGH (Figures $2 \mathrm{~B}$ and $4 \mathrm{~B}$ ). Taken together, the analysis of $\mathrm{CNA}$

and somatic mutations reflected the known complex genetic landscape in those entities. The overall number of somatic lesions in the lymphoma of sister 1 was considerably higher (Figure 2C).

\section{The JAK-STAT pathway has somatic mutations in} multiple genes and is constitutively active

As mentioned above, aCGH revealed a 9p24 gain in both lymphomas (Figure 3A). The amplification of JAK2, a key target of the 9p24 gain, in both tumors was confirmed by FISH (Figure 3B). Moreover, a gain of 12q13 encompassing STAT2 and STAT6, was detected in both lymphomas (Figure 3C). Besides CNA, we also identified somatic mutations in key genes of the JAK-STAT pathway. Each tumor harbored a private missense mutation within the DNA binding domain of STAT6 (Figure 3D). In addition, SOCS1, encoding a negative regulator of the JAK-STAT pathway, was mutated in the lymphoma of sister 1 (Figure 4A). Collectively, these somatic alterations caused constitutive activation of the JAK-STAT pathway as evidenced by high cytoplasmic expression of phosphorylated JAK2 (pJAK2) and increased nuclear pSTAT3 and pSTAT6 expression in most tumor cells (Figure 3E). In summary, despite distinct pathological and clinical features, these data revealed a shared aberrant activation of JAK-STAT signaling which is a known signature in PMBL. ${ }^{10,22,23}$

\section{Genetic alterations related to the distinct clinical outcome}

In contrast to $\mathrm{ABC}$ - and GCB-DLBCL, PMBLs have a favorable prognosis when responding to chemoimmunotherapy. ${ }^{2}$ To investigate genetic lesions associated with the different clinical outcome, we focused on genes with a reported pathogenic role in lymphomas and/or genes which were mutated in more than $10 \%$ of DLBCLs. ${ }^{4-10}$ Mutations in B2M, and TP53, REL and MYC gains as well as a CIITA break apart were confined to the PMBL of sister 1 who died of primary progressive disease (Figure 4A and B). Although amplification of PDL1 result- 
A
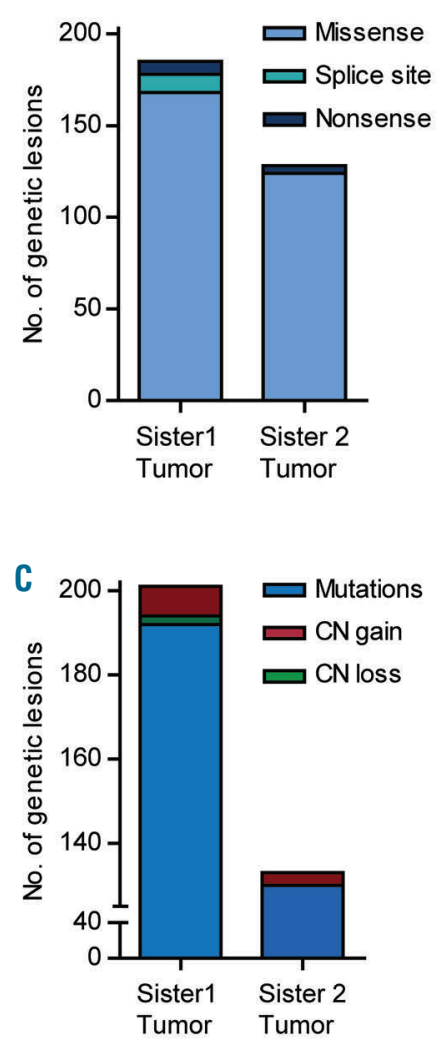

B
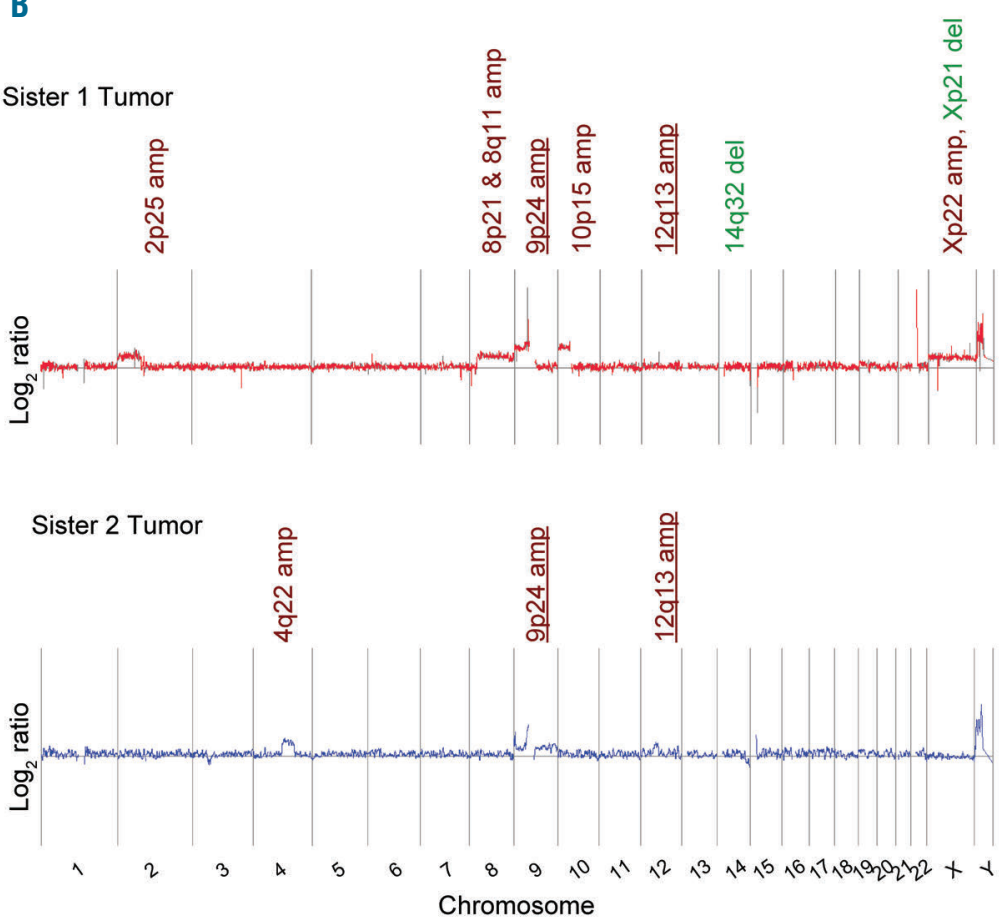

Figure 2. Overall load of numerical and structural genomic alterations in the lymphomas of both siblings. (A) Number (N.) of all validated (by lonProton sequencing) non-silent somatic clonal mutations identified through whole exome sequencing in the two tumors. (B) Chromosomal gains and losses detected in the two lymphomas by array comparative genomic hybridization (aCGH). In the aCGH profiles, the normalized log2 ratios are plotted based on their chromosome position, with vertical bars separating the chromosomes. Regions with losses and gains are represented by decreased and increased log2 ratios, respectively. Genomic changes are marked in red (gain) and green (loss). Copy number (CN) alterations that are present in both tumors are underlined. (C) Combined load of somatic non-silent mutations as well as $\mathrm{CN}$ gains and losses identified in the two investigated lymphomas.

ing from the 9p24 gain (Figure 4A) is a common feature of both lymphomas, PDL1 was only expressed on the malignant B-cells of sister 1 (Figure 4B).

The co-occurrence of genetic alterations involving genes related to immune-cell crosstalk in the lymphoma of sister 1 involving PDL1 expression, B2M p.M1R mutation and genomic alterations of CIITA are an interesting finding that suggests a combined role in escape from immune-surveillance. ${ }^{24,25}$ In summary, we identified genetic lesions that may collectively contribute to the distinct clinical outcome. Of note, TP53 mutations, MYC gains, CIITA translocation and expression of PDL1 on malignant B cells, all solely present in the lymphoma of sister 1 , have been associated with an inferior overall and progression-free survival in DLBCL. ${ }^{26-29}$ BCL2 expression, as observed in the lymphoma of sister 1 , in the absence of a translocation (Online Supplementary Table S2) has a controversial prognostic role. ${ }^{30}$

\section{Whole exome sequencing identified lymphoma risk genes}

The low age- and gender-adjusted incidence rate for sporadic DLBCL $\left(0.1 / 100,000\right.$ cases in Switzerland $\left.{ }^{31}\right)$, and the occurrence of 2 siblings affected by mediastinal B-cell lymphomas suggested a genetic predisposition for lymphomagenesis in this family. Therefore, we performed
WES on DNA from PBMCs of both sisters and all the other members of the core family (Figure 1). A total of 547 rare protein altering variants in 444 genes were identified out of 86,000 screened variants in the germline DNA of each sister. After excluding variants that were present as homozygote in unaffected family members, 274 variants in 234 genes remained. To find a potential link between those 234 candidate genes and deregulated proliferation, we performed a comprehensive gene ontology and pathway enrichment analysis. The result indicated a significant link between 45 of these candidate genes with cancer and/or malignant lymphoma. To identify potential deleterious alterations, the pathogenicity of variants in those 45 cancer related genes was examined by five different in silico algorithms. At the end, 15 variants in 15 candidate genes were predicted as deleterious by all algorithms and were considered for further analyses (Online Supplementary Figure S1A). Mutated genes were significantly enriched in processes like proliferation, lymphocyte activation and response to DNA damage, all pathways crucial for tumorigenesis (Online Supplementary Figure S1B). Of note, MLL the only PMBL/DLBCL susceptibility gene reported so far was not found to harbor variants in this family. ${ }^{19}$

Interestingly, we identified TIRAP among those candidates. TIRAP is an adapter protein that engages signals from TLR2 and 4 and thereby activates the NF-кB path- 
way. Dysregulation of the NF- $\mathrm{KB}$ pathway is the oncogenic hallmark of $\mathrm{ABC}-\mathrm{DLBCL}$ and $\mathrm{PMBL} .{ }^{14}$ Of note, in 420 primary DLBCLs, high TIRAP expression correlated with poor survival and was significantly increased in high risk patients (data generated by SurvExpress ${ }^{32}$ ) (Online Supplementary Figure S2A). Besides, amplifications of $11 \mathrm{q}$ and 11q24 which also contain TIRAP, have been reported in approximately $20 \%$ of DLBCLs and PMBLs. ${ }^{11,33,34}$ Somatic TIRAP mutations occurring in various cancers including DLBCL are listed in COSMIC and genomic sequencing studies on several hundred DLBCLs revealed somatic alterations in TIRAP in roughly $0.5 \%$ of cases. ${ }^{34,35}$ However, the role of TIRAP in tumorigenesis has so far not been investigated. Hence, we functionally investigate whether the identified TIRAP variant in this family contributes to lymphomagenesis.

\section{TIRAP p.R81C variant is a potential novel risk factor for lymphomas}

Whole exome sequencing revealed a heterozygous variant within the coding exon 5 of TIRAP (c.241C>T) in both sisters and their Japanese mother. The variant was absent
A

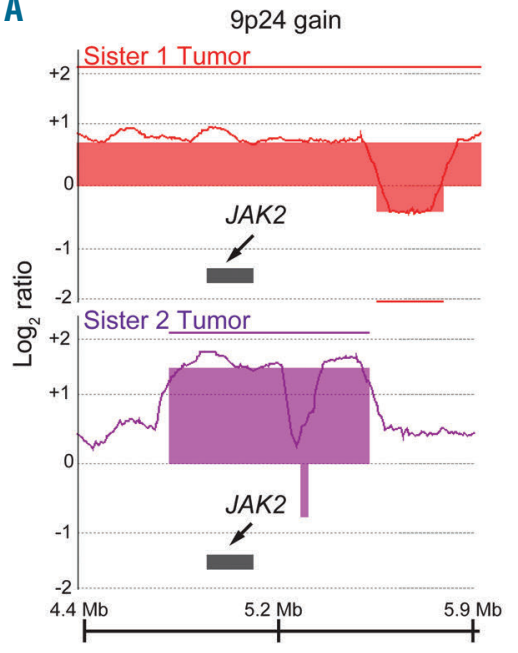

D

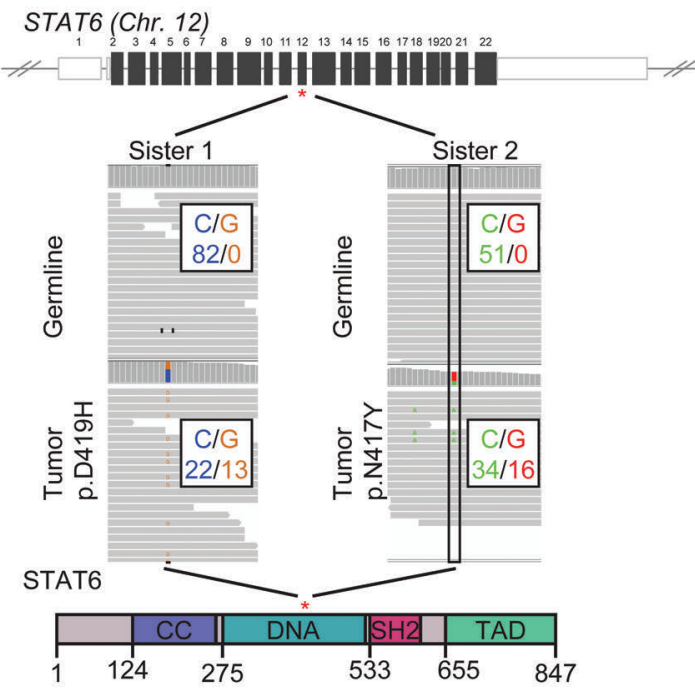

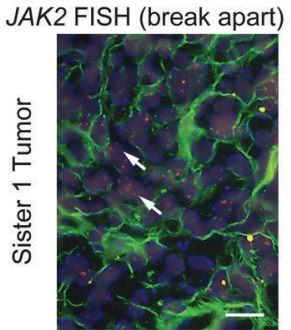

C
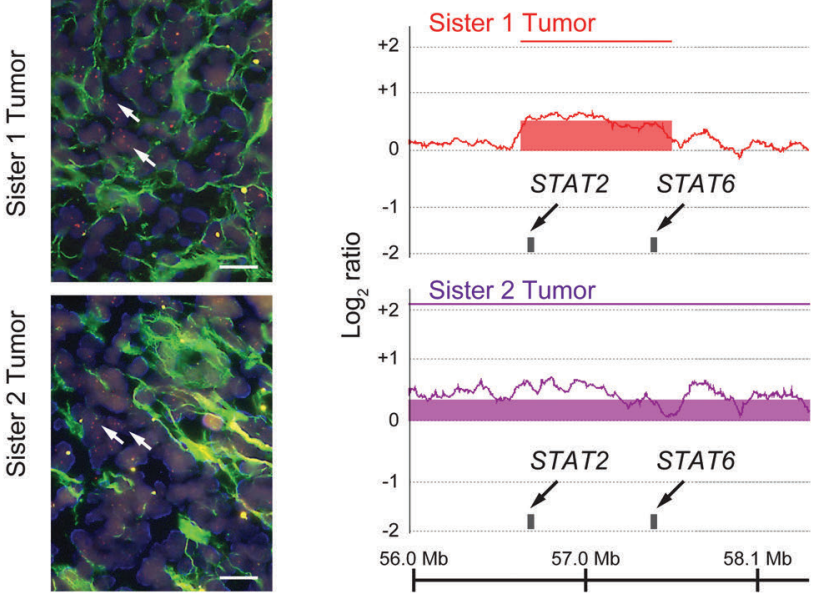

$\mathbf{E}$

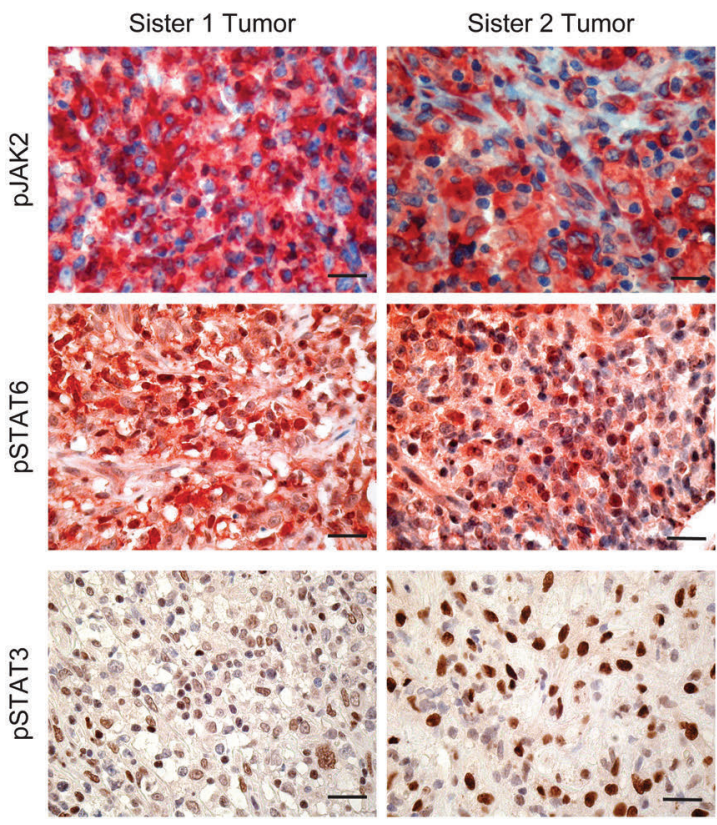

Figure 3. The JAK-STAT pathway has somatic mutations in multiple genes and is constitutively active. (A) aCGH probe view of the $9 \mathrm{p} 24$ gain. A duplication of the JAK2 locus was detected in both samples, shown as an increase of the average log2 ratio above zero (bold line). Shaded area indicated the extent of a copy number aberration. (B) Fluorescence in situ hybridization (FISH) signals with BAC probes for the 5' and 3' regions of JAK2. Arrows indicate examples of cells with multiple FISH signals. Note green autofluorescent sclerosing bundles in the background. Scale bars: $10 \mu \mathrm{m}$. (C) Array comparative genomic hybridization (aCGH) probe view of gains in 12p13 region, which among other genes also affect STAT2 and STAT6 as indicated by arrows. (D) (Top) Schematic representation of the human STAT6 gene locus with open and closed boxes indicating non-coding and coding exons, respectively. (Bottom) Confirmed somatic missense mutations located within the DNA binding domain of STAT6. Protein domain annotation according to Pfam. (E) The expression of phosphorylated (p) pJAK2, pSTAT3 and pSTAT6 in the two lymphomas was assessed by immunohistochemistry. Scale bars: $10 \mu \mathrm{m}$. 
in other unaffected family members (Figure 5A). This variant resulted in a substitution of the arginine at amino acid residue 81 to a cysteine (p.R81C) which is highly conserved among species and located in close proximity to the functional TIR domain (Online Supplementary Figure $S 2 B$ and C). The TIRAP p.R81C variant was predicted to be deleterious by five out of five applied algorithms (Online Supplementary Figure S2D), has a dbSNP identifier (rs138228187) and is reported in COSMIC. It has a global minor allele frequency of 0.00006 and 0.0006 in ExAC and 1000 Genomes, respectively, and 0.0005 in the Japanese population, and is therefore not a polymorphism. ${ }^{36,37}$ Sanger sequencing of cDNA derived from fresh PBMCs of the family members confirmed the TIRAP p.R81C status and expression of the variant allele in sister 2 and her mother (Figure 5A and Online Supplementary Figure S3). Of note, the p.R81C variant was also identified in the lymphomas of both siblings (data not shown).

To investigate the functional consequence of TIRAP p.R81C, we assessed the expression of pIRAK1 and total IRAK4, two downstream kinases and activators of the NF$\kappa B$ signaling pathway. For IRAK4, our analysis was confined to the total protein, as an antibody to reliably determine its phosphorylated form on FFPE tissue was not available. GC B-cells of healthy controls showed little to no pIRAK1 and IRAK4 expression whereas p.R81C TIRAP carrying malignant $\mathrm{B}$-cells of both sisters were clearly positive for these markers (Figure $5 \mathrm{~B}$ and $\mathrm{C}$ ). This suggests that the TIRAP downstream signaling is predominantly active in malignant B-cells with the p.R81C mutation. The relevance of this pathway is underlined by the analysis of 36 primary ABC-DLBCLs that revealed that $63 \%$ and $17 \%$ expressed pIRAK1 and IRAK4, respectively (Figure 5C). GCB-DLBCLs, however, generally lacked the expression of both kinases.

To assess TIRAP/NF- $\mathrm{kB}$ pathway activity in non-malignant cells, we determined the gene expression of TIRAP as well as genes involved in cell proliferation and survival, among them several targets of NF- $\mathrm{kB}$ in PBMCs of living family members. Unsupervised hierarchical clustering analysis revealed two clusters, separating TIRAP p.R81C and wild-type (WT) samples based on the expression signature of selected genes (Figure 5D). PBMCs carrying the TIRAP p.R81C mutation expressed higher levels of TIRAP as well as genes involved in cell survival, cell cycle and proliferation. In contrast, TIRAP WT PBMCs showed higher expression of CASP9, which is implicated in intrinsic apoptosis.

Next, we studied the impact of TIRAP p.R81C on primary B-cells. An increase in proliferating B-cells as deter-
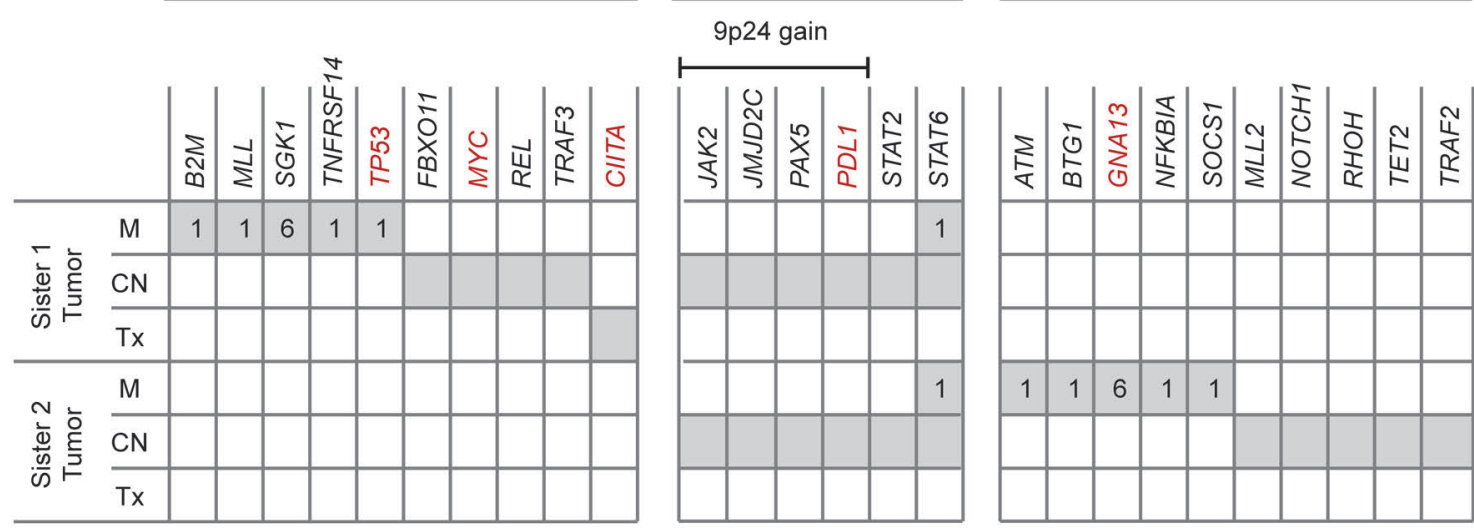

\section{B}
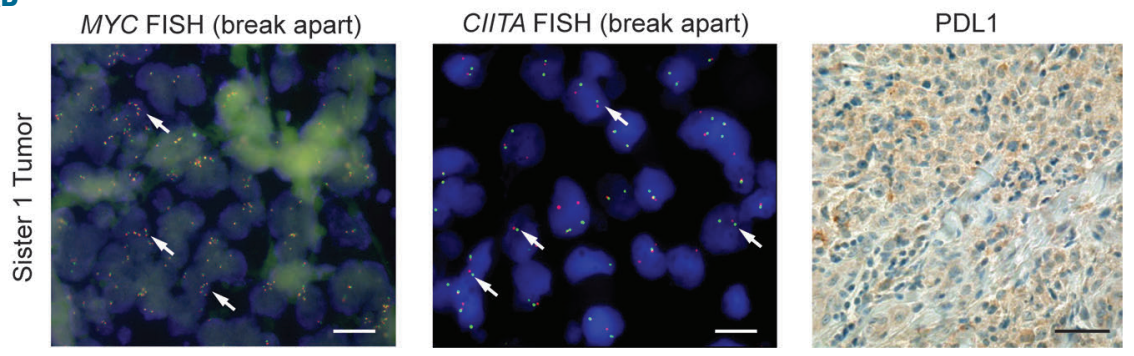

Figure 4. Genetic alterations related to the different clinical outcome. (A) Comparison of the somatic landscape in the two lymphomas implementing the lesions identified by whole exome sequencing, array comparative genomic hybridization (aCGH) and fluorescence in situ hybridization (FISH). Only alterations in genes which are present in more than $10 \%$ of diffuse large B-cell lymphoma (DLBCL) cases and/or with a pathogenic significance in lymphoid malignancies were considered for this comparison. ${ }^{410}$ Mutations (M), copy number alterations (CN) and translocations (Tx) are sorted according to whether they were found to be mutated in both tumors or restricted to one lymphoma only. Numbers indicate the total amount of identified somatic mutations. In red, genes which have been associated with worse clinical outcome..$^{9,26-29}$ (B) (Left) Representative FISH signal patterns using MYC and CIITA break apart assay in the primary mediastinal B-cell lymphoma (PMBL) of sister 1. Arrows indicate examples of cells with MYC (multiple FISH signals) gains and CIITA break apart (split red and green FISH signals), respectively. Scale bars: $10 \mu \mathrm{m}$. (Right) Immunohistochemistry analysis of PDL1 protein expression in the lymphoma of sister 1. Scale bar: $50 \mu \mathrm{m}$. 
A

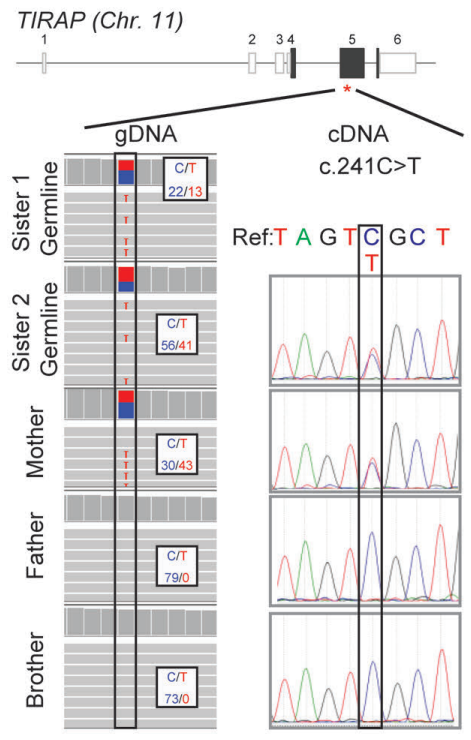

D

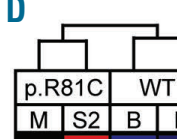

B

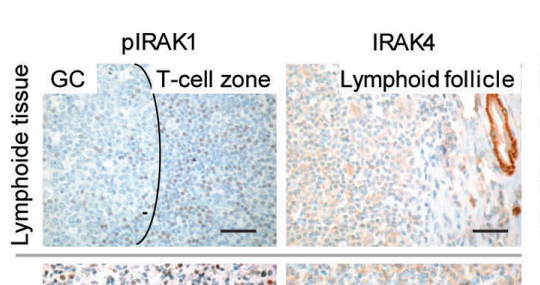

C

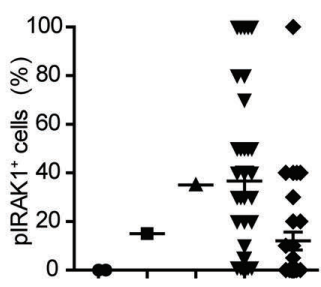

$\bar{s}$
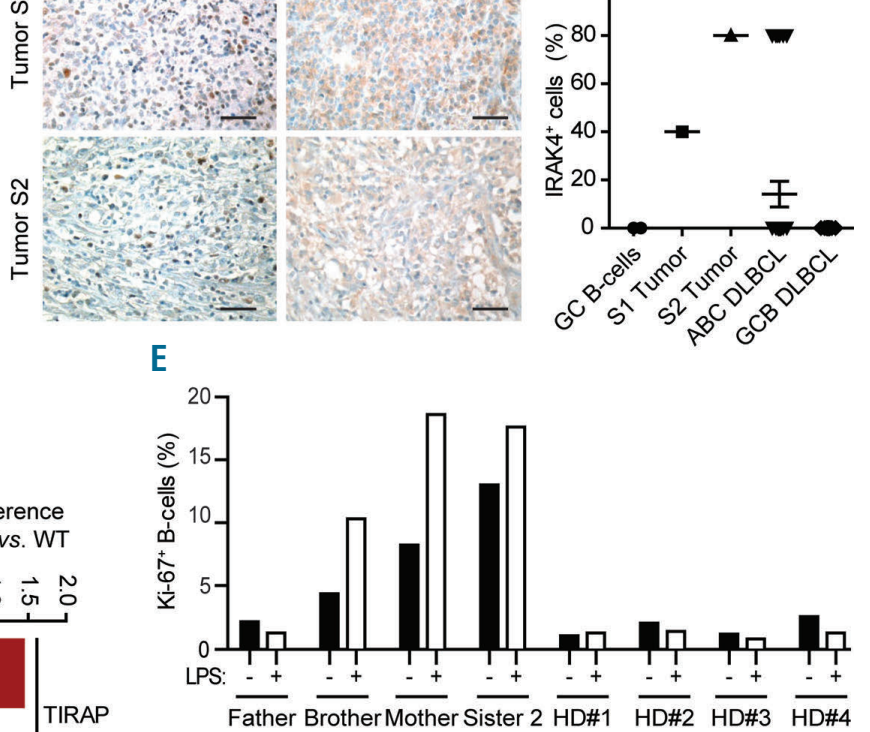

$\overline{\text { Father Brother Mother Sister } 2} \overline{\mathrm{HD \# 1}} \overline{\mathrm{HD \# 2}} \overline{\mathrm{HD \# 3}} \overline{\mathrm{HDH}}$

$\mathrm{F}$

TIRAP wild-type

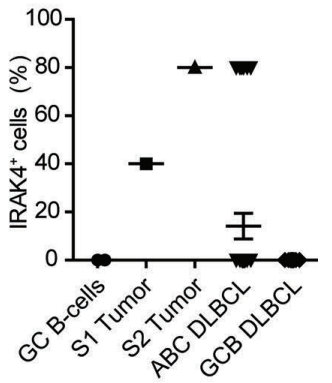

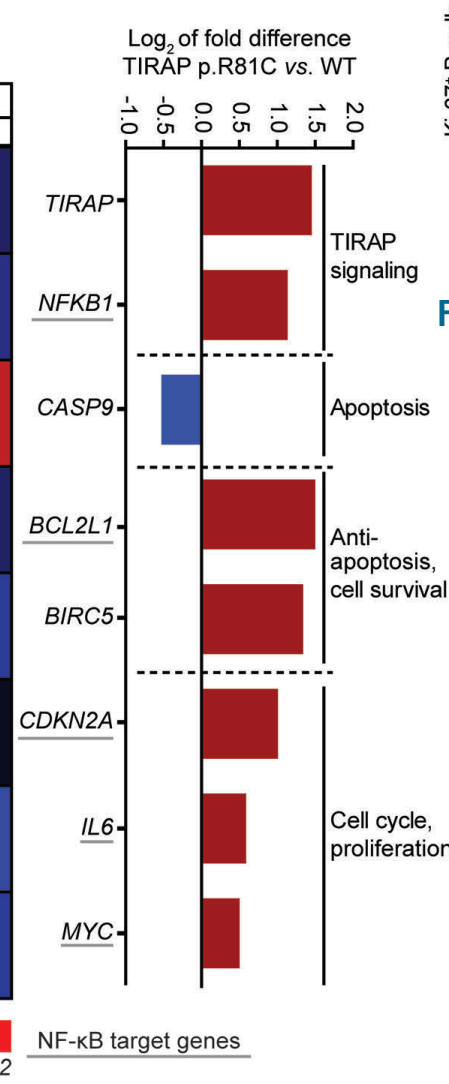
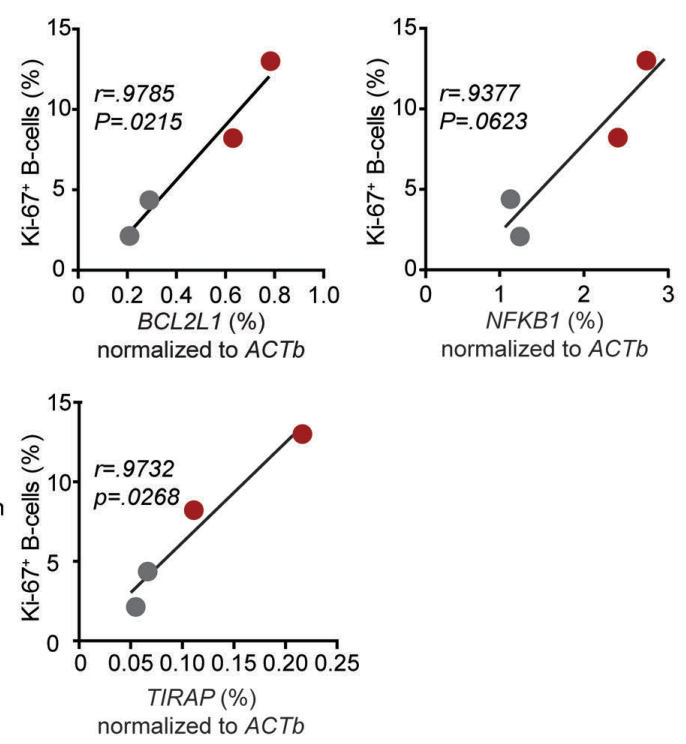

Figure 5. TIRAP p.R81C a potential novel familial lymphoma risk variant. (A) (Top) Schematic representation of the human TIRAP gene locus with open and closed boxes indicating non-coding and coding exons, respectively. (Bottom) Whole exome sequencing data for the affected region of the TIRAP exon 5 visualized in integrative genomic viewer demonstrating a heterozygous variant in both sisters and their mother, whereas homozygous wild-type (WT) sequence was observed in the remaining family members. Sanger sequence data of complementary (c) DNA isolated from fresh peripheral blood mononuclear cells (PBMCs) showing the same variant. Of note, no cDNA was available for sister 1 (deceased). (B) IRAK1 phosphorylation (p) and total IRAK4 expression was assessed by immunohistochemistry (IHC) in the two lymphomas as well as lymphoid tissue of (unmatched) healthy controls. Scale bars: 50 um. (C) Representation of the percentage of cells expressing pIRAK1 and total IRAK4 in samples described in (B) as well as 36 activated B-cell-like diffuse large B-cell lymphoma (ABC-DLBCLs) and 32 germinal center B-celllike diffuse large B-cell lymphoma (GCB-DLBCLs). The primary samples have been described previously..$^{50}$ (D) Heatmap showing hierarchical clustering of mRNA levels of genes involved in NF-KB pathway, cell survival and proliferation in peripheral blood mononuclear cells (PBMCs) of mother (M), sister 2 (S2), brother (B) and father (F). The hierarchical cluster analysis (Euclidean's method) reveals two major clusters representing TIRAP p.R81C mutated and WT individuals. Bar chart showing the log2 fold difference in gene expression in TIRAP p.R81C versus WT family members. (E) PBMCs isolated from family members and age- and gender-matched healthy donors (HD) were cultured in the presence (+) or absence (-) of lipopolysaccharide (LPS) for 12 hours. Ki-67 was measured by flow cytometry on CD20 $0^{+}$B-cells. (F) Linear correlation (Pearson correlation) between Ki-67+ B-cells and BCL2L1 (left), NFKB1 (right) or TIRAP (below) expression in PBMCs as measured by flow cytometry and quantitative PCR (normalized to ACTb), respectively. 
mined by Ki-67 was observed in TIRAP p.R81C compared to TIRAP WT family members (Figure 5E). This was a surprising finding since circulating B-cells are generally quiescent, ${ }^{38,39}$ which we observed in age- and gender-matched healthy individuals as well as TIRAP WT family members. Sanger sequencing confirmed the absence of TIRAP p.R81C variant in healthy donors (Online Supplementary Figure S3). Activation of TLR through lipopolysaccharide (LPS) further enhanced B-cell proliferation, preferentially in TIRAP p.R81C individuals (Figure 5E). The higher level of assessed B-cell proliferation in TIRAP p.R81C cases was positively correlated with the increased gene expression levels of TIRAP, as well as BCL2L1 and NFKB1, as main regulators of NF- $\mathrm{kB}$ signaling in PBMCs of all family members (Figure 5F). Collectively, our data indicate a potential role of p.R81C
A

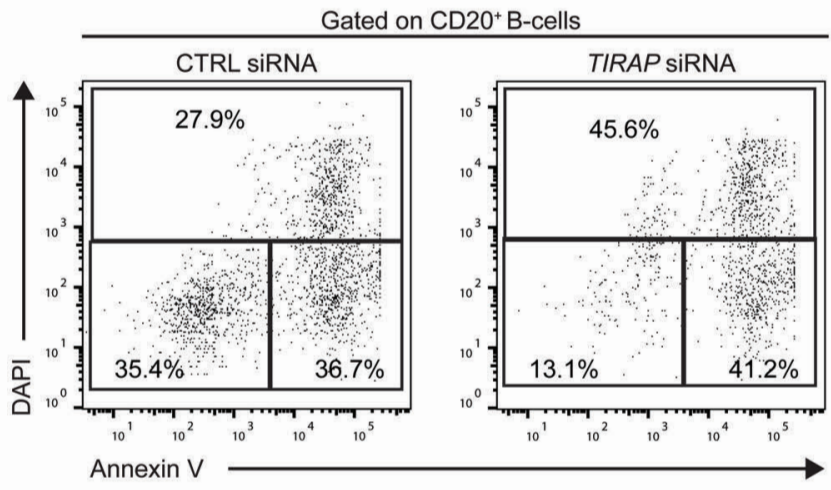

B

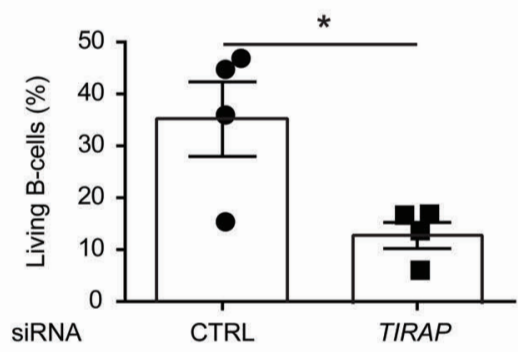

C

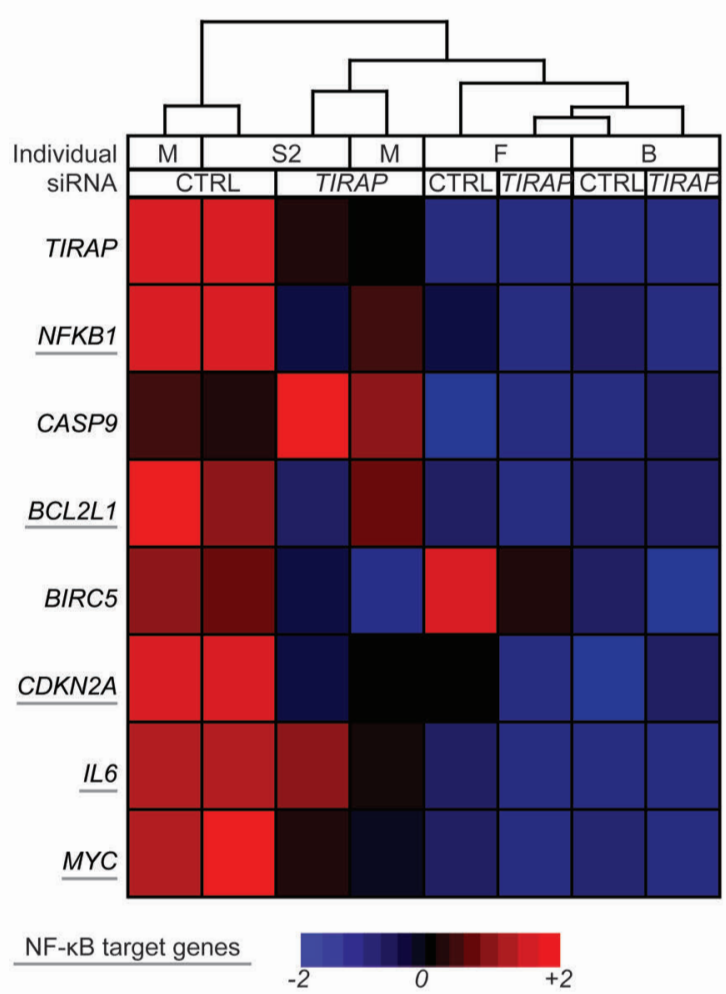

D
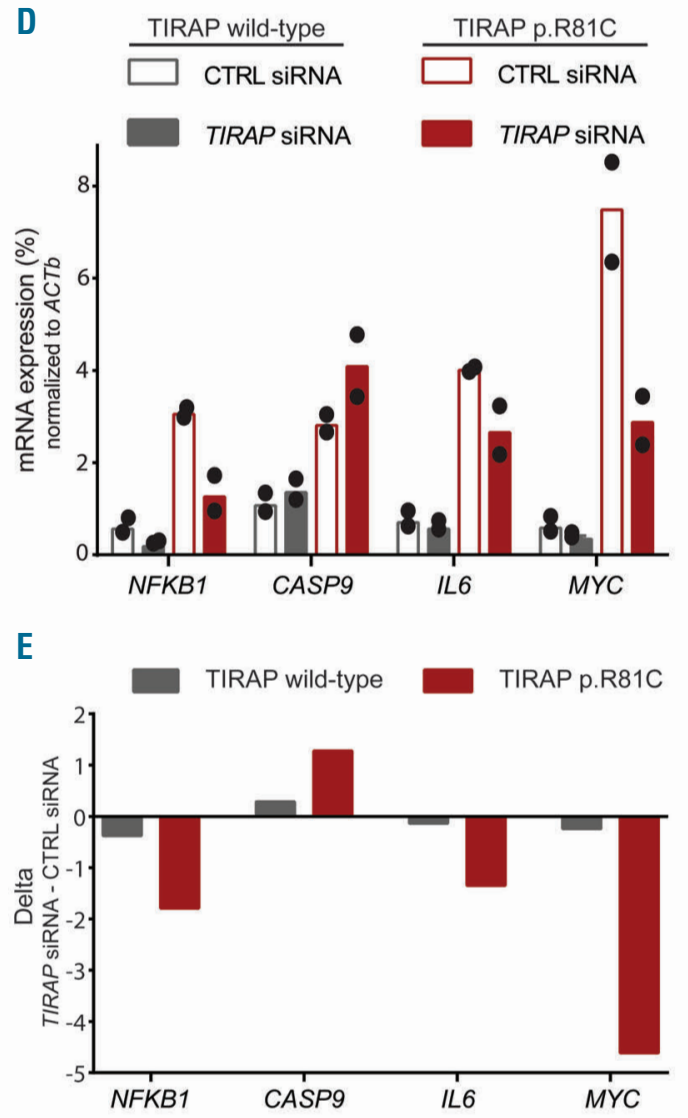

Figure 6. NF-KB signaling mediated by TIRAP is important for B-cell survival. (A) Peripheral blood mononuclear cells (PBMCs) were isolated from family members and transfected with control (CTRL) or TIRAP-directed siRNA. Twenty-four hours following transfection, cell viability of CD20+ B-cells was assessed by Annexin V/DAPI staining and flow cytometry analysis. A representative flow cytometry dot plot and quantification of living B-cells are shown in (A) and (B), respectively. Student $t$-test, ${ }^{*} P<0.05$. (C) Heatmap showing hierarchical clustering of mRNA levels of genes involved in NF-kB pathway, cell survival and proliferation in PBMCs of mother (M), sister $2(\mathrm{~S} 2)$, brother $(\mathrm{B})$ and father $(\mathrm{F})$ treated as described in (A). Data were clustered using standard Euclidean's method based on the average linkage. (D) Bar chart showing the relative expression levels of NFKB1, CASP9, IL6 and MYC genes normalized to ACTb in family members with wild-type (WT) (brother and father) and p.R81C (sister 2 and mother) TIRAP. Dots represent values of each individual of the investigated family. (E) The difference of gene expression in cells transfected with TIRAP or CTRL siRNA was calculated from the mean values shown (D). 
A

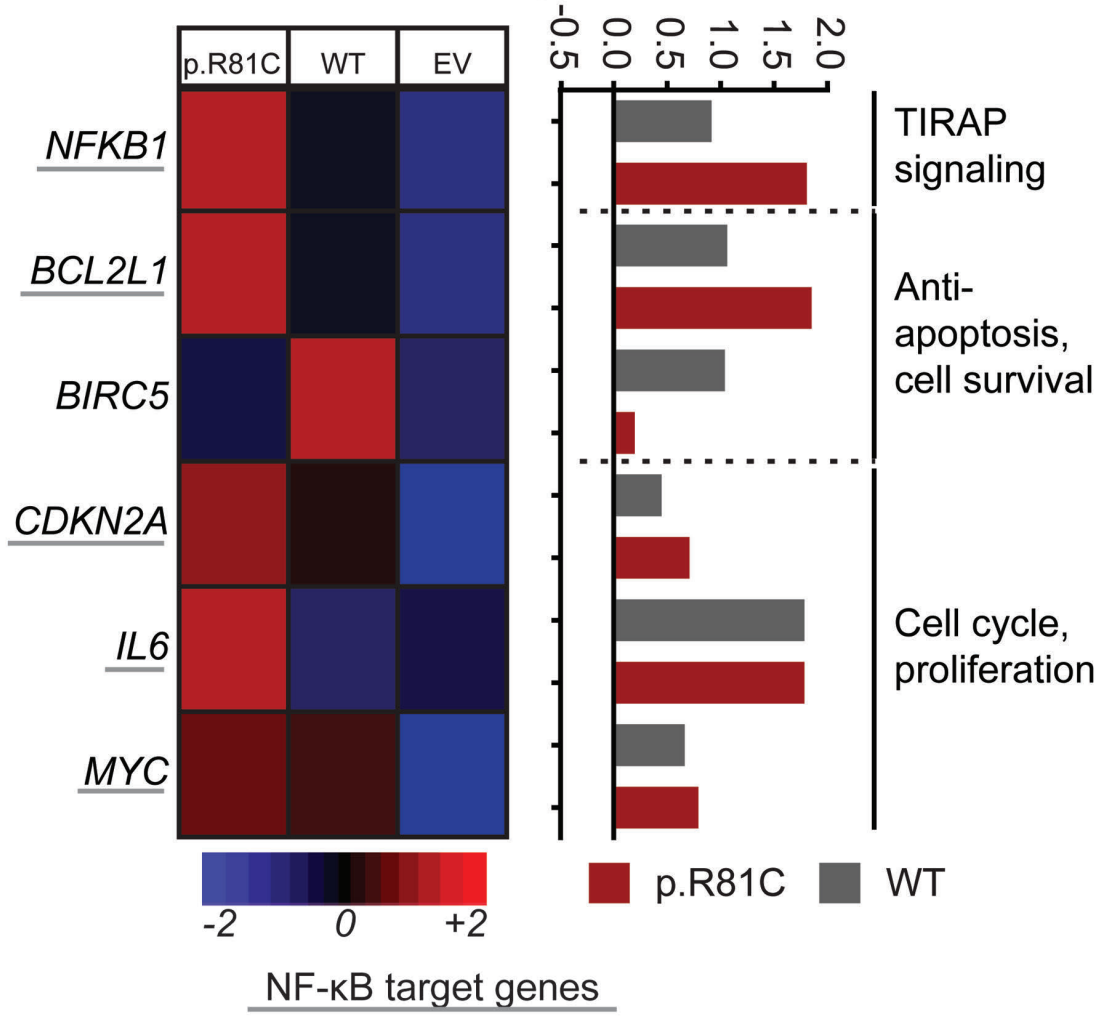

Gated on single cells
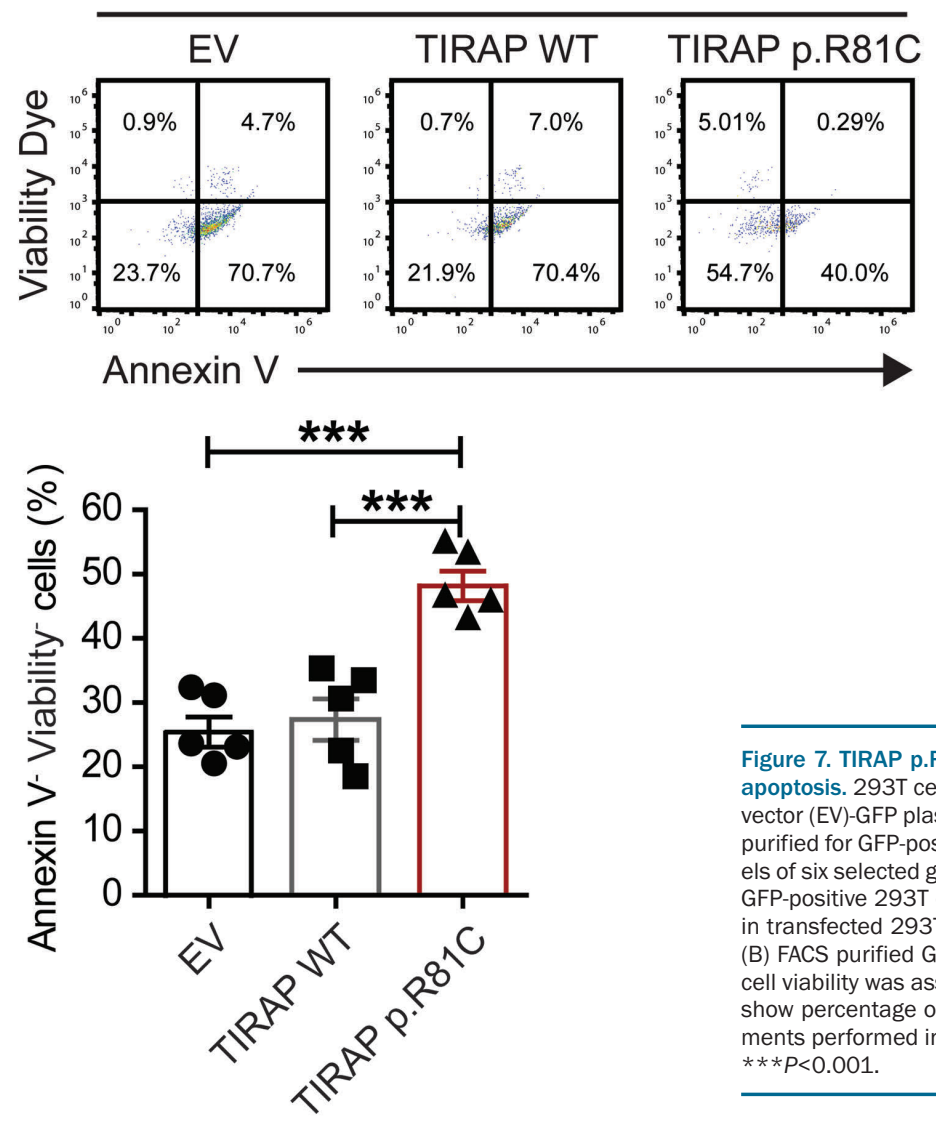

Figure 7. TIRAP p.R81C enhances NF-kB activity and protects against stress-induced apoptosis. 293T cells were transfected with TIRAP WT-GFP, TIRAP p.R81C-GFP or empty vector (EV)-GFP plasmids. Twenty-four hours $(h)$ post transfection, 293T cells were FACS purified for GFP-positive cells. (A) Heatmap showing hierarchical clustering of mRNA levels of six selected genes involved in NF-KB pathway, cell survival and proliferation within GFP-positive 293T cells. Bar chart showing the log2 fold difference of gene expression in transfected 293T cells (TIRAP p.R81C or WT vs. EV) of a representative experiment. (B) FACS purified GFP-positive 293T were cultured in starvation medium for $48 \mathrm{~h}$, and cell viability was assessed by flow cytometry. Bar chart (mean \pm Standard Error of Mean) show percentage of viable cells (AnnexinV ${ }^{-}$and Viability dye-) of 2 independent experiments performed in replicates. One-way ANOVA with Bonferroni post hoc test was used, $* * * P<0.001$. 
variant in activating NF-кB leading to enhanced B-cell proliferation and survival.

\section{INF-אB signaling mediated by TIRAP is important for B-cell survival}

To study the effect of TIRAP on cell survival, we performed a siRNA-mediated knockdown of endogenous TIRAP in PBMCs isolated from available family members. Overall, TIRAP knockdown efficiency was $60 \%$ at the mRNA level (Online Supplementary Figure S4A). TIRAP knockdown significantly diminished the number of living B-cells (Figure 6A and B). This effect was independent of the p.R81C, indicating that TIRAP is an important determinant for B-cell survival. Furthermore, we profiled the expression of genes important for cell survival and proliferation including NF- $\kappa \mathrm{B}$ target genes (Figure $6 \mathrm{C}$ ). Silencing TIRAP strongly reduced the expression of the NF- $\kappa \mathrm{B}$ target genes (NFKB1, IL6 and MYC) in PBMCs, indicating that both WT and p.R81C TIRAP mediate signal through the NF-кB pathway (Figure 6D and Online Supplementary Figure S4). However, downregulation of NF-кB target genes was more pronounced in TIRAP p.R81C PBMCs suggesting that these cells particularly rely on the NF- $\mathrm{kB}$ pathway (Figure 6E and Online Supplementary Figure S4). Consistent with the reduced B-cell survival, CASP9 expression increased following TIRAP silencing in PBMCs to a higher extent in p.R81C mutated cells (Figure 6D and E). Interestingly, NF-кB signature was further reduced following stimulation with LPS (Online Supplementary Figure S5), supporting the concept that TIRAP transduces signals from TLR4. ${ }^{20}$

\section{TIRAP p.R81C drives NF-KB pathway activity and reduces stress-induced cell death}

To evaluate the functional consequence of TIRAP p.R81C, 293T cells were transfected with bidirectional plasmids encoding for EGFP and TIRAP p.R81C, TIRAP WT or empty vector (control), respectively. Under homeostatic conditions, we did not observe any changes in cell viability 24 hours (h) post transfection (Online Supplementary Figure S6). However, gene expression analysis on GFP-positive transfected cells revealed increased expression of the NF- $\mathrm{BB}$ target genes NFKB1, BCL2L1, CDKN2A and MYC by TIRAP p.R81C compared to WT (Figure 7A). Thus, we tested whether these transcriptional changes could affect cell viability upon stress-induced challenge. Therefore, sorted GFP-positive cells were cultured in minimal starving media for $48 \mathrm{~h}$. Surprisingly, cell viability was significantly reduced in control or TIRAP WT transfected cells (Figure 7B). Remarkably, our data indicate that TIRAP p.R81C variant is an upstream activator of NF- $\kappa B$ which leads to a better cell survival/proliferation via enhanced NF- $\mathrm{KB}$ activity and decreased stressinduced cell death.

\section{Discussion}

The etiology of DLBCL is poorly understood. Familial clustering of lymphoma is reported to increase disease risk, indicating a role for genetic factors. ${ }^{15,16}$ Although familial lymphoma cases are rare, studying such pedigrees might identify disease-causing variations and lead to a better understanding of lymphomagenesis. A Finnish family with 3 siblings affected by PMBL and a cousin with extra- nodal DLBCL has been described. ${ }^{19}$ These lymphomas segregate with the p.H1845N mutation in MLL. The role of this variant in lymphomagenesis has not been corroborated by functional studies, and it is to the best of our knowledge the only DLBCL/PMBL predisposing mutation that has been described so far.

Here, we studied a Swiss/Japanese family with 2 sisters affected by B-cell lymphomas in the mediastinum. Although at initial diagnosis their lymphomas were considered, according to the current WHO classification, ${ }^{1}$ as distinct DLBCL subtypes, the characterization of the somatic lesions by WES and aCGH revealed noticeable molecular similarities. Their somatic landscape is marked by multiple alterations affecting important players of the JAK-STAT signaling cascade which collectively lead to constitutive pathway activity, known to be crucially implicated in lymphomagenesis. ${ }^{14}$ Shared gains of 9p24/JAK2 and 12q13 (STAT2 and STAT6) were detected by aCGH. Furthermore, we identified missense hotspot mutations in STAT6 in both lymphomas (p.N417 and p.D417). A significant enrichment of STAT6 mutations in PMBL has been described, with mutations being present in more than $30 \%$ of cases. ${ }^{9,22}$ 9p24/JAK2 gains are also recurrent genetic alterations in $\mathrm{PMBL}$, but are not strictly confined to this subtype. ${ }^{13,40}$

Constitutive activation of the NF- $\mathrm{KB}$ pathway is a hallmark of both ABC-DLBCL and PMBL, and promotes survival of malignant cells. Somatic oncogenic mutations in components of the $\mathrm{B}$-cell receptor signaling pathways including $C D 79 A / B$ and $C A R D 11$ activate NF- $\mathrm{KB} .{ }^{14}$ Gainof-function mutations in MYD88 have been described in $29 \%$ of ABC-DLBCLs. ${ }^{14}$ Moreover, TNFAIP3, which negatively regulates the NF- $\mathrm{\kappa B}$ pathway, is somatically inactivated in one-third of ABC-DLBCLs and PMBLs. ${ }^{14}$ Interestingly, germline mutation in TNFAIP3 and CARD11 have been described in lymphomas complicating primary Sjögren syndrome and congenital B-cell lymphocytosis, respectively. ${ }^{41,42}$ We searched for possible risk alleles associated with the lymphomas in the family investigated by WES, and discovered germline variants in TIRAP and IL1R1 (detailed data on IL1R1 not shown). The latter was not among the final candidate genes, as the homozygous mutation was present in all family members. Nevertheless, the presence of germline variants in two upstream regulators of NF- $\mathrm{\kappa B}$ in a PMBL family is an interesting finding that confirms the importance of the pathway in lymphogenesis. Of note, our data support the cooperation between rare germline variants and constitutive pathway activation in malignant lymphomas. ${ }^{43}$

The predicted damaging effect of TIRAP p.R81C variant occurred at a highly conserved amino acid in close proximity to the functional TIR domain that is stabilized by two internal disulfide bonds. ${ }^{44,45}$ Therefore the substitution of an arginine by a cysteine might have implications on the TIRAP protein interaction with downstream signaling proteins. Of note, an arginine to cysteine mutation in MYD88, another adapter molecule involved in NF-кB signaling, diminished its interaction with TIRAP. ${ }^{46}$

In mice, deletion of Tirap reduced B-cell proliferation in response to TLR4 signaling. ${ }^{20}$ In agreement with this, we observed a direct correlation between B-cell proliferation and TIRAP expression. In this family, a high expression of TIRAP correlated with the p.R81C genotype. In 420 primary DLBLC cases, high TIRAP levels correlated with a poor survival and were significantly increased in high-risk 
DLBCLs. ${ }^{32,47}$ Furthermore, we linked the enhanced proliferation of TIRAP p.R81C B cells with an increased expression of NF-KB target genes and genes involved in cell survival and proliferation in PBMCs. In this context, it is important to stress that we determined the lymphocytes to account for more than $65 \%$ of cells in the PMBC samples analyzed. In addition, TIRAP knockdown was paralleled by a significant decrease in the gene expression signature of the NF- $\kappa \mathrm{B}$ pathway, particularly in PBMCs carrying the p.R81C variant. In contrast, overexpression of TIRAP p.R81C increased NF- $\mathrm{KB}$ gene signature in vitro. Moreover, TIRAP p.R81C-expressing cells showed enhanced resistance to stress-induced apoptosis, indicating that TIRAP p.R81C provides a survival advantage under those conditions.

Our data link TIRAP p.R81C variant/expression with increased B-cell proliferation as well as survival, and thus add TIRAP to the existing network of lymphoma risk genes that are associated with deregulated NF- $\kappa B$ signaling such as TNFAIP3, CD79A/B, MYD88 and CARD11. Interestingly, all these genes were unmutated in the lymphomas of both sisters. Similar to patients expressing the oncogenic p.L265P MYD88 variant, patients with aberrant TIRAP signaling might benefit from IRAK4-selective kinase inhibitors. ${ }^{48}$

Diffuse large B-cell lymphoma is a polygenic disease with a complex pathogenesis. Therefore, additional alterations are required for the full malignant transformation of $\mathrm{B}$-cells. Interestingly, all the family members investigated were found to have a homozygous germline loss of GSTT1, a reported risk factor for lymphomas (Online Supplementary Figure S7). ${ }^{18}$ One can hypothesize that the interplay of the germline TIRAP p.R81C variant and
GSTT1 loss coupled with additional genomic changes culminated in B-cell transformation in the investigated family. The identification of the TIRAP p.R81C variant in a family with mixed ethnic background, along with the demonstration of distinct targets of recurrent mutations in Chinese DLBCLs, ${ }^{49}$ might be an important additional aspect.

Overall, our findings revealed TIRAP p.R81C to be a potential lymphoma risk variant in a family of mixed ethnic background. Our analysis complements the existing view on the different players of the NF- $\mathrm{KB}$ pathway crucially involved in DLBCL.

\section{Acknowledgments}

The authors would like to thank Magne Osteras and his team at Fasteris (Geneva, Switzerland) for their excellent technical assistance. The probe for the TNFAIP3 FISH analysis was a gift from Laura Pasqualucci and Riccardo Dalla-Favera (Institute for Cancer Genetics, Columbia University, New York, NY, USA). The determination of CIITA rearrangement by FISH was kindly performed by Laurence de Leval (Institute of Pathology, Centre Hospitalier Universitaire Vaudois, Lausanne, Switzerland). We thank Lukas Mager, Deborah Krauer, Mario P. Tschan, Emilly Auma, Adrian F. Ochsenbein, Radek Skoda, Federico Santoni, André Schaller, and Outi Kilpivaara for helpful discussions and critical comments.

\section{Funding}

This work was supported by grants from the "Bernische Krebsliga", the "Werner und Hedy Berger-Janser Stiftung", the "Bernische Stiftung für klinische Krebsforschung", the "Stiftung für klinisch-experimentelle Tumorforschung", and a grant from the Inselspital/Bern University Hospital, all to UN.

\section{References}

1. Swerdlow SH, World Health Organization, International Agency for Research on Cancer, editors. WHO classification of tumours of haematopoietic and lymphoid tissues. Revised $4^{\text {th }}$ edition. Lyon: International Agency for Research on Cancer; 2017

2. Rosenwald A, Wright G, Leroy K, et al. Molecular diagnosis of primary mediastinal B cell lymphoma identifies a clinically favorable subgroup of diffuse large B cell lymphoma related to Hodgkin lymphoma. J Exp Med. 2003;198(6):851-862.

3. Savage KJ, Monti S, Kutok JL, et al. The molecular signature of mediastinal large Bcell lymphoma differs from that of other diffuse large B-cell lymphomas and shares features with classical Hodgkin lymphoma. Blood. 2003;102(12):3871-3879.

4. Pasqualucci L, Trifonov V, Fabbri G, et al. Analysis of the coding genome of diffuse large B-cell lymphoma. Nat Genet. 2011; 43(9):830-837

5. Morin RD, Mendez-Lago M, Mungall AJ, et al. Frequent mutation of histone-modifying genes in non-Hodgkin lymphoma. Nature. 2011;476(7360):298-303.

6. Morin RD, Mungall K, Pleasance E, et al. Mutational and structural analysis of diffuse large B-cell lymphoma using whole-genome sequencing. Blood. 2013; 122(7):1256-1265.

7. Lohr JG, Stojanov P, Lawrence MS, et al.
Discovery and prioritization of somatic muta-tions in diffuse large B-cell lymphoma (DLBCL) by whole-exome sequencing. Proc Natl Acad Sci U S A. 2012; 109(10):38793884.

8. Zhang J, Grubor V, Love CL, et al. Genetic heterogeneity of diffuse large B-cell lymphoma. Proc Natl Acad Sci. 2013; 110(4):1398-1403.

9. Dubois S, Viailly P-J, Mareschal S, et al. Next-Generation Sequencing in Diffuse Large B-Cell Lymphoma Highlights Molecular Divergence and Therapeutic Opportunities: a LYSA Study. Clin Cancer Res. 2016;22(12):2919-2928.

10. Gunawardana J, Chan FC, Telenius A, et al. Recurrent somatic mutations of PTPN1 in primary mediastinal B cell lymphoma and Hodgkin lymphoma. Nat Genet. 2014;46(4):329-335.

11. Wessendorf S, Barth TFE, Viardot A, et al. Further delineation of chromosomal consensus regions in primary mediastinal B-cell lymphomas: an analysis of 37 tumor samples using high-resolution genomic profiling (array-CGH). Leukemia. 2007; 21(12):24632469.

12. Joos S, Otaño-Joos MI, Ziegler $\mathrm{S}$, et al. Primary mediastinal (thymic) B-cell lymphoma is characterized by gains of chromosomal material including 9p and amplification of the REL gene. Blood. 1996; 87(4):1571-1578.
13. Lenz G, Wright GW, Emre NCT, et al. Molecular subtypes of diffuse large B-cell lym-phoma arise by distinct genetic pathways. Proc Natl Acad Sci U S A. 2008; 105(36):13520-13525

14. Pasqualucci L, Dalla-Favera R. Genetics of diffuse large B cell lymphoma. Blood. 2018;131(21):2307-2319.

15. Altieri A, Bermejo JL, Hemminki K. Familial risk for non-Hodgkin lymphoma and other lymphoproliferative malignancies by histopathologic subtype: the Swedish Family-Cancer Database. Blood. 2005. 106(2):668-672

16. Goldin LR, Björkholm M, Kristinsson SY, Turesson I, Landgren O. Highly increased familial risks for specific lymphoma subtypes. Br J Haematol. 2009;146(1):91-94.

17. Cerhan JR, Berndt SI, Vijai J, et al. Genomewide association study identifies multiple susceptibility loci for diffuse large B cell lymphoma. Nat Genet. 2014;46(11):12331238.

18. Skibola CF, Curry JD, Nieters A. Genetic susceptibility to lymphoma. Haematologica. 2007;92(7):960.

19. Saarinen S, Kaasinen E, KarjalainenLindsberg M-L, et al. Primary mediastinal large B-cell lymphoma segregating in a family: exome sequencing identifies MLL as a candi-date predisposition gene. Blood. 2013;121(17):3428-3430.

20. Horng T, Barton GM, Medzhitov R. TIRAP 
an adapter molecule in the Toll signaling pathway. Nat Immunol. 2001;2(9):835-841.

21. Li H. Toward better understanding of artifacts in variant calling from high-coverage samples. Bioinformatics. 2014;30(20):28432851.

22. Ritz O, Guiter C, Castellano F, et al. Recurrent mutations of the STAT6 DNA binding domain in primary mediastinal Bcell lymphoma. Blood. 2009;114(6):12361242.

23. Green MR, Monti S, Rodig SJ, et al. Integrative analysis reveals selective 9p24.1 am-plification, increased PD-1 ligand expression, and further induction via JAK2 in nodular sclerosing Hodgkin lymphoma and primary mediastinal large B-cell lymphoma. Blood. 2010;116(17):3268-3277.

24. Mottok A, Woolcock B, Chan FC, et al. Genomic Alterations in CIITA Are Frequent in Primary Mediastinal Large B Cell Lymphoma and Are Associated with Diminished MHC Class II Expression. Cell Rep. 2015;13(7):1418-1431.

25. Challa-Malladi M, Lieu YK, Califano O, et al. Combined Genetic Inactivation of Beta2Microglobulin and CD58 Reveals Frequent Escape from Immune Recognition in Diffuse Large B-cell Lymphoma. Cancer Cell. 2011;20(6):728-740.

26. Xu-Monette ZY, Wu L, Visco C, et al. Mutational profile and prognostic significance of TP53 in diffuse large B-cell lymphoma patients treated with R-CHOP: report from an In-ternational DLBCL Rituximab-CHOP Consortium Program Study. Blood. 2012;120(19):3986-3996.

27. Valera A, López-Guillermo A, CardesaSalzmann $\mathrm{T}$, et al. MYC protein expression and genetic alterations have prognostic impact in patients with diffuse large B-cell lym-phoma treated with immunochemotherapy. Haematologica. 2013;98(10):1554-1562.

28. Steidl C, Shah SP, Woolcock BW, et al. $\mathrm{MHC}$ class II transactivator CIITA is a recurrent gene fusion partner in lymphoid cancers. Nature. 2011;471(7338):377-381.

29. Kiyasu J, Miyoshi H, Hirata A, et al. Expression of programmed cell death ligand 1 is associated with poor overall survival in patients with diffuse large B-cell lymphoma. Blood. 2015;126(19):2193-2201.

30. Visco C, Tzankov A, Xu-Monette ZY, et al. Patients with diffuse large B-cell lymphoma of germinal center origin with BCL2 translocations have poor outcome, irrespective of MYC status: a report from an Internationa DLBCL rituximab-CHOP Consortium Program Study. Haematologica. 2013;98(2): 255-263.

31. Cancer data extracted from the Swiss national dataset managed by the Fondation $\mathrm{Na}$-tional Institute for Cancer Epidemiology and Registration (NICER). Available from: http://www.nicer.org/. Accessed on February 2015. http://www.nicer.org/ de/[Last accessed September 15, 2016.

32. Lenz G, Wright G, Dave SS, et al. Stromal gene signatures in large-B-cell lymphomas. N Engl J Med. 2008;359(22):2313-2323.

33. Bonetti $\mathrm{P}$, Testoni $\mathrm{M}$, Scandurra $\mathrm{M}$, et al. Deregulation of ETS1 and FLI1 contributes to the pathogenesis of diffuse large B-cell ymphoma. Blood. 2013;122(13):2233 2241

34. Chapuy B, Stewart C, Dunford AJ, et al. Molecular subtypes of diffuse large B cell lym-phoma are associated with distinct pathogenic mechanisms and outcomes. Nat Med. 2018;24(5):679-690

35. Schmitz R, Wright GW, Huang DW, et al. Genetics and Pathogenesis of Diffuse Large B-Cell Lymphoma. N Engl J Med. 2018; 378(15):1396-1407.

36. Richards S, Aziz N, Bale S, et al. Standards and Guidelines for the Interpretation of Sequence Variants: A Joint Consensus Recommendation of the American College of Medical Genetics and Genomics and the Association for Molecular Pathology. Genet Med. 2015;17(5):405-424.

37. Higasa K, Miyake N, Yoshimura J, et al Human genetic variation database, a refer ence database of genetic variations in the apanese population. J Hum Genet. 2016; 61(6):547-553.

38. Caraux A, Klein B, Paiva B, et al. Circulating human B and plasma cells. Age-associated changes in counts and detailed characterization of circulating normal CD138- and CD138+ plasma cells. Haematologica. 2010;95(6):1016-1020.

39. Chong $Y$, Ikematsu $H$, Yamaji $K$, et al. CD27(+) (memory) B cell decrease and apopto-sis-resistant CD27(-) (naive) B cell increase in aged humans: implications for age-related peripheral B cell developmental disturbances. Int Immunol. 2005;17(4):383390.
40. Meier C, Hoeller S, Bourgau C, et al. Recurrent numerical aberrations of JAK2 and de-regulation of the JAK2-STAT cascade in lymphomas. Mod Pathol. 2009; 22(3):476-487.

41. Nocturne G, Boudaoud S, Miceli-Richard C, et al. Germline and somatic genetic variations of TNFAIP3 in lymphoma complicating primary Sjögren's syndrome. Blood. 2013;122(25):4068-4076.

42. Snow AL, Xiao W, Stinson JR, et al. Congenital B cell lymphocytosis explained by nov-el germline CARD11 mutations. J Exp Med. 2012;209(12):2247-2261.

43. Yang Y, Schmitz R, Mitala J, et al. Essential role of the linear ubiquitin chain assembly complex in lymphoma revealed by rare germline polymorphisms. Cancer Discov. 2014;4(4):480-493.

44. Valkov E, Stamp A, DiMaio F, et al. Crystal structure of Toll-like receptor adaptor MAL/TIRAP reveals the molecular basis for signal transduction and disease protection. Proc Natl Acad Sci. 2011;108(36):1487914884.

45. Lin Z, Lu J, Zhou W, Shen Y. Structura Insights into TIR Domain Specificity of the Bridging Adaptor Mal in TLR4 Signaling. PloS One. 2012;7(4):e34202

46. Bernuth $\mathrm{H}$ von, Picard $\mathrm{C}$, Jin $Z$, et al Pyogenic Bacterial Infections in Humans with MyD88 Deficiency. Science. 2008; 321(5889):691-696

47. Aguirre-Gamboa $\mathrm{R}$, Gomez-Rueda $\mathrm{H}$ Martínez-Ledesma E, et al. SurvExpress: an online biomarker validation tool and database for cancer gene expression data using survival analysis. PloS One. 2013;8(9):e74250.

48. Kelly PN, Romero DL, Yang Y, et al. Selective interleukin-1 receptor-associated kinase 4 inhibitors for the treatment of autoimmune disorders and lymphoid malignancy. J Exp Med. 2015;212(13):21892201

49. Miranda NFCC de, Georgiou K, Chen L, et al. Exome sequencing reveals novel mutation targets in diffuse large B-cell lym phomas derived from Chinese patients. Blood. 2014:124(16):2544-2553.

50. Juilland M, Gonzalez M, Erdmann T, et al CARMA1- and MyD88-dependent activation of Jun/ATF-type AP-1 complexes is a hallmark of $\mathrm{ABC}$ diffuse large $\mathrm{B}$-cell lymphomas. Blood. 2016;127(14):1780-1789. 